\title{
L'acte concernant les écoles d'industrie (1869) : une mesure de prophylaxie sociale dans un Québec en voie d'urbanisation
}

\section{Renée Joyal}

Volume 50, numéro 2, automne 1996

URI : https://id.erudit.org/iderudit/305509ar

DOI : https://doi.org/10.7202/305509ar

Aller au sommaire du numéro

Éditeur(s)

Institut d'histoire de l'Amérique française

ISSN

0035-2357 (imprimé)

1492-1383 (numérique)

Découvrir la revue

Citer cette note

Joyal, R. (1996). L'acte concernant les écoles d'industrie (1869) : une mesure de prophylaxie sociale dans un Québec en voie d'urbanisation. Revue d'histoire de l'Amérique française, 50(2), 227-240. https://doi.org/10.7202/305509ar d'utilisation que vous pouvez consulter en ligne. 


\title{
NOTE DE RECHERCHE
}

\section{L'ACTE CONCERNANT \\ LES ÉCOLES D'INDUSTRIE (1869) \\ UNE MESURE DE PROPHYLAXIE SOCIALE DANS UN QUÉBEC EN VOIE D'URBANISATION ${ }^{1}$}

\author{
RENÉE JOYAL \\ Département des sciences juridiques \\ Université du Québec à Montréal
}

Adopté par la Législature de Québec en 1869, l'Acte concernant les écoles d'industrie ${ }^{2}$ a souvent été présenté comme l' «ancêtre» de la Loi sur la protection de la jeunesse $e^{3}$ actuelle. L'examen des deux textes législatifs fait toutefois ressortir des fondements, des objectifs et des modalités d'application radicalement différents. Il est donc difficile d'apercevoir une quelconque filiation entre la loi de 1869 et celle de 1977, ce qui ne veut pas dire, par ailleurs, que ces deux textes ne soient aucunement apparentés. L'Acte concernant les écoles d'industrie constitue, en effet, la première intervention spécifique du législateur québécois à l'égard de l'enfance abandonnée.

Avant cette date, en effet, il faut chercher ailleurs que dans des dispositions légales les principaux mécanismes de solution des problèmes familiaux. La famille élargie, le voisinage, la communauté villageoise et les institutions de charité jouent alors à cet égard un rôle primordial, le tout sous l'œil attentif de l'Église et du clergé ${ }^{4}$. Sauf

1. Ce texte a été élaboré et rédigé dans le cadre d'un projet de recherche subventionné par le Conseil de recherches en sciences humaines du Canada; maître Carole Chatillon et madame Louise Chauvette, M.A. (Histoire), ont largement contribué à la recherche documentaire.

2. Acte concernant les écoles d'industrie, SQ, 1869, ch. 17.

3. Loi sur la protection de la jeunesse, LQ, 1977, ch. 20.

4. Nive Voisine, en collaboration avec André Beaulieu et Jean Hamelin, Histoire de l'Église catholique au Québec (1608-1970) (Montréal, Fides, 1971), 57.

RHAF, vol. 50, $\mathrm{n}^{\circ}$ 2, automne 1996 
quelques exceptions visant des problèmes particuliers ${ }^{5}$, les rapports entre parents et enfants ne sont touchés que par les lois générales de police et d'assistance. Le Code civil du Bas-Canada, adopté en 1866, consacre la notion de puissance paternelle héritée de la Coutume de Paris, mais ne prévoit aucun mécanisme de contrôle de cette institution.

Ce n'est pas par hasard que la question de l'enfance abandonnée trouve un écho législatif particulier en 1869. Première réponse étatique à un problème que les remèdes traditionnels n'arrivent plus à juguler, le texte alors adopté reflète les préoccupations des élites politiques et religieuses de l'époque et traduit une nouvelle forme de collaboration entre elles.

\section{1) LE CONTEXTE DE L'INTERVENTION LÉGISLATIVE}

Les changements qui, en cette seconde moitié du XIX ${ }^{\mathrm{e}}$ siècle, affectent la société québécoise, comme d'autres sociétés avant elle, ainsi que les moyens mis en œuvre au sein de plusieurs de celles-ci pour remédier aux problèmes nouveaux suscités par cette évolution ont un impact direct sur le législateur de 1869.

\section{A) La société québécoise}

À cette époque, qui est celle de l'entrée du Québec dans la Confédération, la population de la province dépasse le million d'habitants. Montréal et Québec sont devenues des agglomérations importantes, avec respectivement 100000 et 55000 habitants environ. Ce mouvement d'urbanisation, lié à l'industrialisation accélérée qui caractérise cette période, se répercute de diverses façons sur la vie familiale et la situation des enfants.

Bien qu'on ne puisse parler d'industrialisation massive pour le Québec avant le $\mathrm{XX}^{\mathrm{e}}$ siècle, une économie capitaliste y est cependant en formation, fondée sur l'établissement de manufactures locales et l'afflux d'une main-d'œuvre immigrante en provenance notamment de l'Irlande ${ }^{6}$. À part quelques exceptions - chemins de fer, bois -, les industries légères qui se développent alors, notamment dans les domaines de l'alimentation, du vêtement, de la chaussure et du tabac,

5. Voir, par exemple, en matière de vagabondage: Acte pour remédier plus efficacement à divers abus préjudiciables à l'amélioration de l'agriculture, et à l'industrie dans cette province, et pour d'autres objets, Statuts provinciaux du Bas-Canada, 1824, ch. 33, art. 30.

6. Stanley B. Ryerson, Le capitalisme et la Confédération (Montréal, Éditions Parti-Pris, 1972), 235. 
font largement appel au travail des femmes et des enfants ${ }^{7}$. S'il a été clairement établi qu'un grand nombre de tout jeunes enfants ont été embauchés dans des manufactures à cette époque, il demeure que la main-d'œuvre juvénile était surtout composée d'adolescents et de jeunes gens ${ }^{8}$. Le sweating system se répand rapidement: il permet, en effet, aux employeurs d'épargner sur les frais de production et d'écarter toute velléité d'association ou de syndicalisation". Dans l'ensemble, les conditions de travail sont pénibles; durant les crises économiques et les saisons mortes, le chômage jette une multitude de travailleurs et leurs familles dans la misère et le désarroi ${ }^{10}$.

La situation du logement urbain est critique. La proportion de locataires s'accroît sensiblement à Montréal, qui mérite alors pleinement sa réputation de «ville de locataires». Dans les quartiers ouvriers, les logements sont surpeuplés et les conditions sanitaires déplorables. $\mathrm{Au}$ milieu du siècle, personnes et animaux se côtoient dans les agglomérations urbaines. Nombreux sont les ménages qui, pour améliorer leurs moyens de subsistance, élèvent poules, vaches et même cochons, sans compter les milliers de chevaux qui assurent le transport des personnes et des marchandises. Les odeurs de la pollution industrielle se mêlent à celles du fumier, des égouts et des carcasses d'animaux en décomposition ${ }^{11}$. Montréal connaît alors un taux de mortalité infantile extrêmement élevé: plus de un enfant sur quatre n'atteint pas l'âge de un an. L'impureté de l'eau et du lait, de même que l'insuffisance des mesures de vaccination contre la variole et la diphtérie en auraient été les causes principales ${ }^{12}$.

À la situation critique du logement urbain et aux carences de l'hygiène publique s'ajoute l'absence totale de mesures ou de prestations sociales. L'aide aux indigents s'appuie exclusivement sur la charité privée, notamment sur les institutions religieuses - crèches, orphelinats, hospices - qui se sont développées au cours du $\mathrm{XIX}^{\mathrm{e}}$ siècle, s'ajoutant aux hôpitaux généraux fondés dès les débuts de la colonie. Les secours distribués par les conférences de Saint-

7. D. Suzanne Cross, «La majorité oubliée: le rôle des femmes à Montréal au $19^{e}$ siècle», dans Marie Lavigne et Yolande Pinard, dir., Travailleuses et féministes. Les femmes dans la société québécoise (Montréal, Boréal Express, 1983), 70-71.

8. Bettina Bradbury, Familles ouvrières à Montréal. Âge, genre et survie quotidienne pendant la phase d'industrialisation (Montréal, Boréal, 1995), 14.

9. Idem, 179.

10. Jean Hamelin et Yves Roby, Histoire économique du Québec (1851-1896) (Montréal, Fides, 1971), 307.

11. B. Bradbury, Familles ouvrières à Montréal..., 46 et 179.

12. Terry Copp, Classe ouvrière et pauvreté. Les conditions de vie des travailleurs montréalais 1892-1929 (Montréal, Boréal Express, 1978), 23, 105 et 107. 
Vincent-de-Paul, les multiples activités déployées entre autres par les Sœurs Grises et les Sœurs de la Providence contribuent aussi à soulager ces misères ${ }^{13}$.

Mais les remèdes appliqués dans le cadre de la société agricole et artisanale du début du siècle sont vite dépassés dans les agglomérations urbaines importantes que sont devenues Montréal et Québec: avec l'accroissement et la diversification de la population, les formes traditionnelles de solidarité s'y affaiblissent. Lorsque, aux difficultés liées à ces nouvelles conditions de vie, s'ajoutent le chômage, la maladie ou le décès de l'un ou des parents, la misère qui s'ensuit jette de nombreux enfants à la rue.

\section{B) Des remèdes observés ailleurs}

Confrontée à ce phénomène du vagabondage des enfants, l'Angleterre du XIX ${ }^{\mathrm{e}}$ siècle décide de les soumettre à un système d'éducation préventive. C'est en 1857 que le Parlement britannique adopte la première Industrial Schools Act dont l'objectif était le placement dans des écoles spéciales des enfants mineurs âgés de sept à quatorze ans «qui vagabondaient et risquaient de mal tourner en raison de la négligence parentale et des mauvaises influences de leur milieu ${ }^{14} \gg$.

$\mathrm{Au} \mathrm{XIX}^{\mathrm{e}}$ siècle, on note aussi, aux États-Unis comme en France, le développement de colonies pénitentiaires ou d'institutions spécialisées où se retrouvent, à côté des enfants délinquants qui en constituent la clientèle première, un grand nombre d'enfants errants, livrés au vagabondage ou à la prostitution ${ }^{15}$. Des institutions privées s'apparentant à des écoles industrielles recueillent, notamment dans les Etats de la côte est américaine, des vagabonds, des enfants pauvres, maltraités ou menacés de déchéance morale ${ }^{16}$.

13. Voir, à ce sujet, l'étude abondamment documentée de Huguette Lapointe-Roy, Charité bien ordonnée. Le premier réseau de lutte contre la pauvreté à Montréal au 19 e siècle (Montréal, Boréal, 1987).

14. Mario Provost, «Le mauvais traitement de l'enfant. Perspectives historiques et comparatives de la législation sur la protection de la jeunesse», Revue de droit de l'Université de Sherbrooke, 22 (1991): 30.

15. Voir, pour les États-Unis, Danielle Laberge, «L'invention de l'enfance: modalités institutionnelles et support idéologique», Criminologie, 18 (1958): 73; pour la France, Philippe Robert, Traité de droit des mineurs. Place et rôle dans l'évolution du droit français contemporain (Paris, Cujas, 1969), 76-77, 104 et ss., et Michelle Perrot, «L'enfance révolutionnée par la Révolution? Parents et enfants au XIX ${ }^{\mathrm{e}}$ siècle», dans M.-F. Lévy, dir., L'enfant, la famille et la Révolution française (Paris, Olivier Orban, 1990), 404.

16. H. Folks, The Care of Destitute, Neglected and Delinquent Children (Washington, National Association of Social Workers, 1978), 224-226. Réimpression de l'édition originale de 1902), cité par M. Provost, «Le mauvais traitement...», 32. 
D'autre part, ce n'est qu'en 1874 que l'Ontario se dote d'une loi établissant des écoles d'industrie sur son territoire. Il faut toutefois noter que, par la suite, l'évolution des lois ontariennes de protection de l'enfance sera beaucoup plus rapide qu'au Québec. Cette particularité tient largement à la mise sur pied de sociétés de protection de l'enfance dès les années 1880 en Ontario; de tels organismes n'apparaissent au Québec que beaucoup plus tard, en 1922 dans la communauté anglophone, mais seulement à partir des années 1930 dans le milieu canadien-français où les institutions de charité dirigées par des communautés religieuses recueillent un grand nombre d'enfants malheureux.

\section{C) Des facteurs de changement}

L'on constate d'abord, sous le régime de l'Union, l'adoption de deux lois particulières concernant les personnes mineures délinquantes. La première, l'Acte concernant le mode de juger et punir les jeunes délinquants ${ }^{17}$, adoptée en 1857 , établit une procédure sommaire dans le cas de personnes de moins de seize ans accusées de «simple larcin» ou d'une infraction analogue. Elle prévoit la possibilité de détenir les personnes mineures condamnées, dans la prison commune ou dans une «maison de correction». La seconde, l'Acte pour établir des prisons pour les jeunes délinquants, adoptée la même année et complétée en $1858^{18}$, prévoit les modalités d'incarcération des personnes mineures dans des «prisons de réforme» qui leur sont exclusivement réservées.

Ces deux textes témoignent du souci du législateur d'établir un régime particulier de traitement des personnes mineures délinquantes, tant au point de vue de la procédure que de la peine. Les termes employés, «maisons de correction», «prisons de réforme», illustrent le passage de l'idée de châtiment à celle de redressement dans ce contexte particulier ${ }^{19}$. Cette évolution se situe dans le cadre plus large de la «spécialisation» des institutions d'assistance et de répression au

17. Acte concernant le mode de juger et punir les jeunes délinquants, SC, 1857, ch. 29 .

18. Acte pour établir des prisons pour les jeunes délinquants, SC, 1857, ch 28; SC, 1858 , ch. 88 .

19. La même observation est faite à l'égard de l'évolution de la situation en France au $\mathrm{XIX}^{\mathrm{e}}$ siècle, notamment par Philippe Meyer, L'enfant et la raison d'État (Paris, Seuil, 1977), 60; dans les faits, toutefois, ces «maisons de correction» qu'on a voulu établir à partir d'une loi de 1799 en ce sens, ne sont mises sur pied que plus tard et ne se distinguent guère des prisons communes: Jacques Laplante, Prison et ordre social au Québec (Ottawa, Presses de l'Université d'Ottawa, 1989), 59. 
cours du XIX ${ }^{\mathrm{e}}$ siècle $^{20}$. Paradoxalement, c'est pour satisfaire ces deux visées qu'avait été mise sur pied à Montréal, en 1818, une maison d'industrie où jeunes et adultes livrés à l'oisiveté et à la petite délinquance étaient hébergés et affectés à divers travaux. Il s'agissait là d'une entreprise de prévention du vagabondage et de la mendicité demandée par les élites locales et dotée d'une reconnaissance légale par la Chambre d'Assemblée. Organisée de manière à s'insérer dans l'appareil de police de la ville, mais ne disposant toutefois d'aucune subvention gouvernementale, l'institution doit fermer ses portes après quelques années d'existence. À Québec, quelques actions temporaires du même ordre sont entreprises dans les années qui suivent. Finalement, en 1836, on tente à nouveau d'établir des maisons d'industrie à Montréal et à Québec. Dans cette ville, l'édifice qui abrite les indigents est détruit par un incendie quelques mois plus tard et les choses en demeurent là. À Montréal, la maison cesse ses opérations moins d'un an après sa mise sur pied, incapable de se financer sans l'aide du gouvernement, qui lui fait défaut ${ }^{21}$. Par la suite, cependant, des communautés religieuses établissent des refuges du même type destinés entre autres aux jeunes filles en difficultée 22 .

Enfin, peu avant la présentation du texte de loi qui nous occupe, il convient de signaler le dépôt à l'Assemblée de nombreuses pétitions émanant surtout de personnalités ecclésiastiques de Montréal - notamment les révérends Baile, supérieur des Sulpiciens, et Ramsay, aumônier de La Miséricorde, et de Québec - notamment le très révérend Taschereau (il s'agit vraisemblablement du grand vicaire du diocèse de Québec, futur archevêque et cardinal) réclamant l'adoption d'une telle loi ${ }^{23}$. L'hébergement de personnes mineures, filles ou garçons, dans des institutions spécialisées posait en effet un problème d'ordre légal. Les autorités des refuges déjà mis sur pied ou qu'on avait l'intention de créer ne pouvaient recevoir ces enfants qu'avec le consentement de leur père, titulaire de la puissance paternelle. En l'absence de ce consentement, seul un ordre du tribunal pouvait donner un caractère légal à leur action: d'où la nécessité d'une loi en ce sens $^{24}$. Ainsi s'expliquent les pressions exercées sur le législateur par les autorités ecclésiastiques. On se rappelle qu'à l'époque, la très

20. La diversification des modes de régulation des comportements participe de la logique capitaliste déjà à l'œuvre à l'époque de l'Union, selon Jean-Marie Fecteau, Un nouvel ordre des choses: la pauvreté, le crime, l'État au Québec, de la fin du XVIII siècle à 1840 (Montréal, VLB éditeur, 1989), 264.

21. Idem, 189 et ss.

22. Voir, à ce sujet, l'étude très fouillée de Jacques Laplante, Prison et..., 77 et ss.

23. Débats de l'Assemblée législative, 1867-1870 (reconstitution), 171, 192, 204 et 211.

24. Voir, à ce sujet, Alice Parizeau, Protection de l'enfant: échec? (Montréal, Presses de l'Université de Montréal, 1979), 9. 
grande majorité des institutions d'assistance était dirigée, en milieu catholique, par des communautés religieuses.

\section{2) LES CARACTÉRISTIQUES DU TEXTE DE LOI}

Interrogé par des membres du Parlement sur les objectifs poursuivis par le gouvernement, l'Honorable Pierre-Joseph-Olivier Chauveau, alors premier ministre ${ }^{25}$, explique que le bill vise à protéger les enfants et à leur éviter les dangers qui conduisent à une vie immorale ${ }^{26}$. Voyons comment ces objectifs se traduisent dans le texte de loi.

\section{A) Les enfants visés}

L'Acte concernant les écoles d'industrie ne s'intéresse qu'aux enfants de moins de quatorze ans, aucun âge minimal n'y étant par ailleurs fixé. On peut cependant penser que cette loi a été appliquée dans les faits surtout aux enfants d'âge scolaire, les enfants abandonnés en très bas âge étant plutôt recueillis à l'orphelinat ou à la crèche en vue d'une adoption ou d'un placement auprès d'une famille. Le texte vise essentiellement les enfants «trouvés errants» ou en compagnie de «voleurs de profession», les orphelins et les enfants dont le père a été condamné à une peine de prison ou de travaux forcés et qui, pour cette raison, sont «sans moyens d'existence». Il mentionne également les enfants «réfractaires» ou que leurs parents sont «incapables de maîtriser».

Cette première intervention législative traduit clairement les préoccupations des autorités publiques à l'égard d'enfants dont la situation ou le comportement les placent sur la voie de la délinquance. C'est d'abord l'ordre social, mis à mal par le vagabondage et la petite délinquance qui se développent dans les agglomérations urbaines, qu'on cherche à préserver par l'adoption de cette loi. Les mécanismes mis en place à cette fin relèvent du même état d'esprit.

\section{B) Les mécanismes prévus}

Toute personne peut conduire devant deux juges de paix ou un magistrat l'enfant qui se trouve dans l'une des situations énumérées

25. L'Honorable Pierre-Joseph-Olivier Chauveau fut également le premier titulaire du ministère de l'Instruction publique créé en 1867 et aboli en 1876. Peu avant sa nomination, il avait visité plusieurs pays européens en vue d'étudier leurs systèmes scolaires: Louis-Philippe Audet, «Le premier ministère de l'Instruction publique au Québec 1867-1876», Revue d'histoire de l'Amérique française, 22,2 (septembre 1968): 176-177. Il avait sans doute pu observer le fonctionnement des premières écoles d'industrie mises sur pied en Angleterre après l'adoption de l'Industrial Schools Act de 1857.

26. Débats de l'Assemblée législative, 1867-1870 (reconstitution), 218. 
par la loi; toutefois, dans le cas d'un enfant «réfractaire» ou «incontrôlable», c'est le directeur de l'institution de charité ayant recueilli cet enfant ou même son propre père, beau-père ou tuteur qui peut solliciter à son égard l'intervention de l'autorité publique. Après la tenue d'une enquête sommaire, les juges de paix ou le magistrat saisis de l'affaire peuvent, s'ils l'estiment à propos, ordonner le placement de l'enfant dans une école d'industrie.

Une telle école d'industrie peut être reconnue officiellement sur demande faite par ses directeurs au lieutenant-gouverneur, qui charge alors l'inspecteur désigné à cette fin «de s'enquérir de la condition de l'école, et si elle est en état de recevoir les enfants...» Sur réception d'un rapport satisfaisant, le secrétaire de la province délivre un certificat à l'école concernée, qui sera par la suite sujette à une inspection annuelle.

Les écoles d'industrie sont tenues d'instruire et d'élever les enfants qu'elles consentent à recevoir, ainsi que de pourvoir à leur subsistance. Ces écoles ne relèvent pas du département de l'Instruction publique mais du secrétaire de la province. Les programmes scolaires y sont établis selon la bonne volonté et les ressources des personnes qui les dirigent. C'est seulement à partir de 1945 et surtout 1946 que les programmes d'études des écoles d'industrie devront être préparés et appliqués sous l'autorité du Conseil de l'instruction publique $^{27}$.

L'ordonnance de placement constitue un ordre de détention pour une durée déterminée - la période prévue ne doit cependant pas dépasser l'époque à laquelle l'enfant atteint l'âge de seize ans - , et le fait de quitter l'école sans autorisation est considéré comme une évasion et, dans certains cas, puni comme tel. Après une certaine période d'hébergement, l'enfant peut être autorisé à loger à l'extérieur ou être placé en apprentissage, le tout sous la supervision des autorités de l'école. Des dispositions particulières favorisent le respect des croyances religieuses de l'enfant et son instruction dans la religion à laquelle il appartient.

Le financement de ces institutions est réparti entre le trésor provincial et les municipalités concernées. S'ils en ont les moyens, les parents sont tenus à une contribution et l'on exige une somme plus importante s'ils ont eux-mêmes demandé «l'internement» de l'enfant.

27. Marie-Paule Malouin, dir., L'Univers des enfants en difficulté au Québec entre 1940 et 1960 (Montréal, Bellarmin, 1996), 178. 


\section{C) Les limites de l'intervention de l'État}

Ce qui se dégage du texte de loi, c'est que l'État intervient pour suppléer la famille, lorsqu'elle n'existe pas ou qu'elle a délaissé l'enfant, ou encore pour renforcer l'autorité du père de famille ou du directeur de l'institution. Il ne cherche pas à contrôler ou à modifier l'exercice de la puissance paternelle; il vise plutôt la formation et le redressement de l'enfant par l'imposition d'un cadre disciplinaire rigide et l'apprentissage d'un métier.

Selon un rapport des inspecteurs de prisons daté de 1870, «[les] Écoles d'industrie, retirant de l'abandon ou du vice, ces jeunes créatures, souvent douées de grâce ou de dons précieux, les instruisant, formant les cœurs à la vertu, et les habituant au travail, en feront, au lieu de coupable, qu'elle eût été, une classe utile, industrieuse et morale ${ }^{28}$ 》.

Il est utile de rappeler ici que l'Acte concernant les écoles d'industrie a été adopté au même moment que l'Acte concernant les écoles de réforme $e^{29}$, ce qui illustre l'étroite association que les parlementaires québécois - à l'instar de leurs collègues britanniques qui avaient eux aussi légiféré sur les deux questions à quelques années d'intervalle - faisaient entre le placement en école de réforme, qui visait le redressement des jeunes délinquants et le placement en école d'industrie, dont le but était de prévenir la délinquance chez les enfants errants ou abandonnés. D'ailleurs, en pratique, les deux clientèles seront souvent confondues et abritées dans les mêmes locaux. En effet, même si en vertu de la loi les écoles de réforme ne peuvent recevoir que des jeunes condamnés pour un délit, il demeure qu'elles sont confiées, tout comme les écoles d'industrie, à des communautés religieuses, contrairement aux «prisons de réforme» de 1858. C'est le même souci de «moralisation» qui préside au nouvel ordonnancement créé par l'adoption des deux lois.

\section{3) LA SUITE DES ÉVÉNEMENTS}

Entre le moment de son adoption, en 1869, et celui de son abrogation, en 1950, l'Acte concernant les écoles d'industrie fut plusieurs fois modifié. Avant d'examiner le sens de ces remaniements, relatons le développement institutionnel auquel la nouvelle loi a donné lieu.

28. Document cité par Jacques Laplante, Prison et..., 89.

29. Acte concernant les écoles de réforme, SQ, 1869, ch. 18. 


\section{A) Les institutions mises sur pied}

Nous avons déjà observé plus haut que, dans le contexte de la diversification des institutions d'assistance amorcée au cours du $\mathrm{XIX}^{\mathrm{e}}$ siècle, certaines communautés religieuses établissent des refuges de «protection» et de «préservation» pour «jeunes filles en danger».

À cette fin, les sœurs du Bon-Pasteur d'Angers ouvrent à Montréal, à partir de 1844 , le refuge du Bon-Pasteur. Un an après l'adoption des lois de 1869, elles mettent sur pied, à Saint-Hubert, une institution qui sert à la fois d'école de réforme et d'industrie. Bien que les mêmes bâtiments servent aux deux types d'écoles, il semble que les différentes classes y aient été séparées les unes des autres ${ }^{30}$. À Québec, les sœurs du Bon-Pasteur, qui ont mis sur pied l'asile Sainte-Madeleine en 1850, fondent, en 1870, l'hospice Saint-Charles, à la fois école de réforme et d'industrie.

Quant aux jeunes garçons délinquants, ils peuvent, à partir de 1858, être détenus dans la «prison de réforme» établie à l'îleaux-Noix, puis à Saint-Vincent-de-Paul, et, finalement, en 1873, dans un ancien hospice construit par le philanthrope Olivier Berthelet. La direction en est alors confiée aux frères de la Charité. Aux jeunes délinquants s'ajoutent, surtout depuis 1869 , les enfants placés en école d'industrie par les juges de paix ou le magistrat et il semble que, dans les faits, il n'y ait pas eu de distinction entre les deux clientèles ${ }^{31}$.

Dans un ouvrage publié en 1876, Pierre-Joseph-Olivier Chauveau fait le point sur les écoles de réforme et les écoles d'industrie. Ces institutions, écrit-il, «sont confiées, à Montréal, aux frères de la Charité et aux sœurs du Bon-Pasteur, et à Québec et Lévis, aux sœurs du Bon-Pasteur et aux sœurs de la Charité. Ces communautés religieuses, instituées dans un but de charité, sont devenues des communautés enseignantes; elles ont un grand nombre d'écoles et de pensionnats dans les campagnes. L'école de réforme protestante est établie à Sherbrooke ${ }^{32} \gg$.

Quelque soixante ans plus tard, alors que la population du Québec a plus que triplé, on y dénombre six écoles d'industrie accueillant 2242 enfants et cinq écoles de réforme qui en hébergent 859. La vocation respective des deux types d'écoles semble donc s'être précisée avec le temps. Toutefois, on ne peut certes pas parler d'étanchéité.

30.. Jacques Laplante, Prison et..., 81.

31. Idem, 87-88.

32.. Pierre-Joseph-Olivier Chauveau, L'instruction publique au Canada (Québec, Imprimerie Augustin Roy et Cie, 1876), 295-296. 
À preuve, le Girls Cottage and Industrial School de Sweetsburg, qui figure sur la liste des écoles de réforme telle qu'établie par CharlesÉdouard Bourgeois, alors une sommité dans le domaine ${ }^{33}$.

\section{B) Les amendements législatifs}

Il serait fastidieux de relater toutes et chacune des modifications apportées au texte de loi original. Certaines méritent toutefois d'être signalées, car elles témoignent des hésitations du législateur qui, soumis à des pressions contradictoires, limite, d'une part, l'intervention de l'État dans le domaine et, d'autre part, étend l'application de la loi à d'autres catégories d'enfants.

La loi de 1869 s'applique, on s'en souvient, aux enfants de moins de quatorze ans. Cet âge maximal est abaissé à douze ans en 1884, en même temps qu'est formulée la règle voulant qu'aucun ordre d'«internement» en école d'industrie ne puisse être donné à l'égard d'un enfant de moins de sept ans ${ }^{34}$. Abaissé à quatre ans en $1890^{35}$, cet âge minimal est ramené à six ans en $1892^{36}$, alors que l'âge maximal est rehaussé à quatorze ans en $1894^{37}$.

En ce qui a trait au financement des écoles d'industrie, on peut observer le même souci de préciser et de restreindre la responsabilité de l'État. Les modifications adoptées reflètent par ailleurs des tensions entre les municipalités et les autorités provinciales à ce sujet. Le texte de 1869 n'est pas très explicite à propos du financement des écoles: on y mentionne la participation financière des parents et des municipalités où sont domiciliés les enfants, mais c'est sur le trésor de la province que semble reposer la survie des écoles. En 1884, les maires, avec l'autorisation de leur conseil, sont autorisés à demander le placement de diverses catégories d'enfants en école d'industrie; au même moment, on impute la moitié du coût d'entretien des enfants placés en école d'industrie aux municipalités, à charge pour elles de se pourvoir contre les parents ou l'enfant, s'ils ont des biens. En outre, l'«internement» de l'enfant à la demande de ses parents est désormais à la

33. Charles-Édouard Bourgeois, Une richesse à sauver: l'enfant sans soutien (Trois-Rivières, Éditions du Bien public, 1947), 241 et 256.

34. Acte pour amender l'acte 32 Victoria, chap. 17, concernant les écoles d'industrie, SQ, 1884, ch. 23.

35. Acte amendant l'article 3142 des Statuts refondus de la province de Québec concernant les écoles d'industrie, SQ, 1890, ch. 28. Il est toutefois précisé dans ce texte que les enfants de quatre à neuf ans peuvent être «envoyés et détenus» dans l'orphelinat agricole de Notre-Dame de Montfort!

36. Loi concernant les écoles d'industrie, SQ, 1892, ch. 29.

37. Loi amendant la loi des écoles d'industrie, SQ, 1894, ch. 32. 
charge exclusive de ceux-ci. En 1892, la responsabilité financière des municipalités atteint les trois quarts du coût d'entretien des enfants placés et même la totalité à l'égard de certains enfants négligés. La proportion de la contribution municipale est ramenée à la moitié en 1894, exception faite de situations particulières, et, à compter de cette date, les maires n'ont plus besoin de l'autorisation du conseil municipal pour demander l' «internement» de certains enfants. On ne peut s'empêcher de noter que cette répartition des frais entre les municipalités et le trésor provincial préfigure en quelque sorte les règles de partage des dépenses d'assistance publique qui seront établies à partir de 1921.

D'autre part, le texte de loi intègre progressivement d'autres catégories d'enfants. Ainsi, à partir de 1884, son application s'étend aux enfants qui ont besoin de protection en raison de la maladie continuelle, de l'extrême pauvreté, de l' «ivrognerie» ou des «habitudes vicieuses» de leurs parents. Viennent s'y ajouter, en 1894, les enfants «infirmes» et ceux dont les parents ne sont pas jugés «dignes» d'en prendre soin. En 1912, on ajoute à cette nomenclature déjà considérable l'enfant «habituellement battu» ou «traité cruellement» par ses parents ou les personnes chez qui il réside ${ }^{38}$. Cette préoccupation grandissante à l'égard des enfants maltraités et négligés dans le cadre familial se traduit, en Grande-Bretagne, en Ontario et dans plusieurs États américains par l'adoption de lois de protection de l'enfance s'appuyant sur l'existence de sociétés locales de sauvegarde ${ }^{39}$. Au Québec, elle ne débouche cependant pas sur des mesures différentes de celle préconisée en 1869, soit le placement des enfants concernés en école d'industrie. Il faut attendre les années 1930 et 1940 pour que se dessinent certaines modalités d'intervention de l'État à l'intérieur de la sphère familiale ${ }^{40}$.

\section{CONCLUSION}

Première intervention spécifique de l'État à l'égard des enfants livrés à eux-mêmes, l'Acte concernant les écoles d'industrie se situe aux confins de la réaction sociale à la délinquance. On a vu plus haut l'association faite par les parlementaires entre les enfants envoyés à l'école de réforme et ceux placés en école d'industrie. On sait égale-

38. Loi amendant les Statuts refondus, 1909, concernant les jeunes délinquants [sic], SQ, 1912, ch. 39.

39. M. Provost, Le mauvais traitement..., 37 et ss.

40. Voir, à ce sujet, Renée Joyal et Carole Chatillon, «La loi québécoise de protection de l'enfance de 1944, genèse et avortement d'une réforme», Histoire sociale/Social History, 53 (1994): 33 et ss. 
ment que, dans les faits, les deux clientèles sont souvent accueillies et traitées de la même manière.

Il n'est donc pas étonnant que, en 1912, on ajoute au texte de loi une disposition ${ }^{41}$ qui prévoit que certains groupes d'enfants - fugueurs, désobéissants, incontrôlables, se livrant à la paresse, tenant une conduite immorale, un langage obscène ou indécent puissent être considérés comme de jeunes délinquants et poursuivis en vertu de la Loi sur les jeunes délinquants ${ }^{42}$, qui vient d'être adoptée par le Parlement fédéral en 1908. À l'inverse, dans certaines circonstances, la nouvelle loi fédérale permet à l'autorité provinciale de transformer une «affaire de délinquance» en «affaire de protection» et donc de continuer selon les règles de la législation provinciale les procédures entamées en vertu de la législation fédérale à l'égard des enfants concernés ${ }^{43}$. Les deux textes sont, jusqu'à un certain point, des vases communicants. De plus, dans la foulée de l'adoption de la nouvelle loi fédérale, un tribunal spécialisé, la Cour des jeunes délinquants, est créé dans la province, d'abord à Montréal, en 1910, puis à Québec, en 1940. Ce tribunal est habilité, à partir de 1912, à entendre les poursuites intentées en vertu tant de la Loi des écoles d'industrie que de la Loi sur les jeunes délinquants, ce qui est certes de nature à favoriser l'interpénétration des deux lois.

Entre les jeunes délinquants et ceux qui, dans l'esprit des élites, sont exposés à le devenir, il n'y a donc pas de différence marquée ${ }^{44}$. Tous ces jeunes sont sur une pente dangereuse et il convient de pourvoir à leur redressement. C'est l'ordre public qui est en jeu ici et c'est une œuvre de moralisation qu'on entreprend pour en assurer la sauvegarde et prévenir le désordre. Ce qui est visé, ce n'est pas l'acte posé mais l'attitude, le comportement, le style de vie. «Le délinquant, écrit Michel Foucault, se distingue de l'infracteur par le fait que c'est moins son acte que sa vie qui est pertinente pour le caractériser ${ }^{45} . » \mathrm{Ce}$

41. Voir la note 43.

42. Loi concernant les jeunes délinquants, SC, 1908, ch. 40.

34. Voir, à ce sujet, Jean Trépanier, «Principes et objectifs guidant le choix des mesures prises en vertu de la Loi sur les jeunes contrevenants», Revue du Barreau, 49 (1989): 563; Jean Trépanier et Françoise Tulkens, Délinquance et protection de la jeunesse. Aux sources des lois belge et canadienne sur l'enfance (Bruxelles/Montréal/Ottawa, De Boeck/Presses de l'Université de Montréal/Presses de l'Université d'Ottawa, 1995), 43-44.

44. Les légistes, par ailleurs, semblent avoir fait une distinction entre les deux problématiques, puisque, dans les Statuts refondus de 1888, l'Acte concernant les écoles de réforme figure sous le titre VII, «De la police et du bon ordre. De la sûreté et de la santé publique», alors que l'Acte concernant les écoles d'industrie apparait sous le titre VIII, «Des associations charitables, philanthropiques et de prévoyance». On retrouve la même division dans les Statuts refondus de 1909. 255 .

45. Michel Foucault, Surveiller et punir, Naissance de la prison (Paris, Gallimard, 1975), 
regard particulier porté sur les mœurs d'une classe sociale vue comme dangereuse explique pour une large part les interventions «prophylactiques» du législateur québécois de 1869 à l'endroit des enfants qui en sont issus et annonce déjà le mouvement de défense sociale qui inspirera les politiques des gouvernements fédéral et provincial face aux jeunes en difficulté à la fin du $\mathrm{XIX}^{\mathrm{e}}$ et au début du $\mathrm{XX}^{\mathrm{e}}$ siècle. 